\title{
EXPECTATIVAS LABORALES DE LOS CENTENNIALS PARA LOS NEGOCIOS TURÍSTICOS DE CANCÚN. ENFOQUE BASADO EN LA TEORÍA DE LAS NECESIDADES DE MCCLELLAND
}

\author{
RICARDO SONDA DE LA ROSA \\ rsonda@ucaribe.edu.mx \\ DAMAYANTI ESTOLANO CRISTERNA \\ destolano@ucaribe.edu.mx \\ SANDRA GUERRA MONDRAgÓN \\ sguerra@ucaribe.edu.mx \\ Universidad del Caribe
}

La creciente participación de trabajadores conocidos como centennials, obliga a los negocios turísticos a implementar nuevas estrategias para reclutar y retener a su personal. Este trabajo identifica las expectativas laborales bajo la teoría de las necesidades de McClelland, desde un enfoque cuantitativo de alcance correlacional, utilizando el análisis factorial de componentes, a través de la aplicación de una encuesta a la población estudiantil próxima a egresar, del programa educativo de Turismo Sustentable y Gestión Hotelera de la Universidad del Caribe, ubicada en Cancún, Quintana Roo, México. Los hallazgos declarados como motivadores fueron la infraestructura, el balance de vida, el clima laboral y los programas de sustentabilidad característicos de esta generación.

Palabras clave: centennials, contratación, expectativas, negocio turístico.

\section{CENTENNIALS JOB EXPECTATIONS FOR CANCUN TOURISM BUSINESSES. APPROACH BASED ON MCCLELLAND'S THEORY OF NEEDS}

The growing participation of young workers known as centennials, compels tourism businesses to implement new strategies to recruit and retain their staff. This paper identifies labor expectations under McClelland's theory of needs, from a quantitative approach of correlational scope using the factorial analysis of components, applied through a survey to the student population about to graduate from the educational program of Sustainable Tourism and Hotel Management at the Universidad del Caribe, located in Cancun, Quintana Roo, Mexico. The findings declared as motivators are infrastructure, life balance, work environment and sustainability programs hallmarks of this generation.

Keywords: centennials, recruitment, expectations, tourist business.

Fecha de recepción: 15 de julio de 2020 Fecha de aceptación: 9 de octubre de 2020.

CÓMO CITAR: Sonda, R., Estolano, D. y Guerra, S. (2020). Expectativas laborales de los centennials para los negocios turísticos de Cancún. Enfoque basado en la teoría de las necesidades de McClelland. Dimensiones Turísticas, 4(7), 151-180. https://doi.org/10.47557/KJLU44449 


\section{Introducción}

as diferencias y confrontaciones entre generaciones, en aspectos sociales y culturales, han sido siempre una constante en el entorno laboral; sin embargo, se han agudizado en los últimos años debido a que, por primera vez en la historia contemporánea, las organizaciones emplean hasta cuatro de ellas en un mismo contexto empresarial (Bennett et al., 2012; Costanza et al., 2012; Kuron et al., 2015).

Los denominados Baby boomers, Generación X (o xennials), Generación Y (más conocidos como millennials), y ahora los Generación Z (también llamados centennials), en conjunto, desarrollan una equilibrada fuerza de trabajo en muchos negocios turísticos, cada uno con motivaciones que es útil determinar, para distintos fines.

Se designa como centennials a los jóvenes que nacieron a mediados de los años 90 y hasta el 2005, en una época caracterizada por lo digital (Magallón, 2016). Autores como Boschma y Groen (2008) los describen con atributos fortalecidos en el sentido de la autenticidad, el crecimiento personal, la rapidez y la socialización. Bajo este paradigma, tales individuos se incorporan hoy en día al mercado, de forma gradual, razón por la que se ha hablado respecto a sus motivaciones y expectativas.

Autores como Guillot-Soulez y Soulez (2014), Golik (2013), Ng et al. (2010), y Smith (2010), coinciden en que estos perfiles buscan prioritariamente un equilibrio entre su vida personal y el desempeño profesional; motivación que deben contemplar las empresas en busca de resultar atractivas para ellos.

Wilton (2008) plantea que los centennials, antes de ingresar en una organización, anteponen variables como el desarrollo de habilidades, proyectos de vida y retos innovadores, por encima del factor retribución económica. Según Smith (2010), esta generación es la primera en reducir las diferencias entre hombres y mujeres; además, se les considera incluyentes y abiertos a otras preferencias de género, distintas a las conservadoras.

Thompson y Gregory (2012) afirman que estas actitudes han tenido impacto en los procesos tradicionales de selección, reclutamiento y contratación, mismos que requieren evolucionar a corto y mediano plazo, a fin de que disminuyan los índices de rotación. Esto parece demandar el establecimiento de estrategias de prospección diversas para los nuevos colaboradores (Al-Emadi et al., 2015; Kultalahti y Viitala, 2015; Rani y Samuel, 2016). 
Moreno et al. (2015) describen que el mayor índice de rotación se presenta durante los primeros seis meses después de incorporar al empleado a una organización. Esto ocasiona cuantiosos costos en el proceso de reclutamiento, selección, contratación, inducción y capacitación al puesto; además, provoca una percepción negativa de los demás trabajadores y supervisores, traducida en incertidumbre, cansancio, frustración y estrés, a causa de la baja. Esta situación puede detonar un efecto dominó y hacer que los colaboradores de planta evalúen sus alternativas, en busca de otro espacio para la obtención de una estabilidad mayor.

El índice de rotación es multifactorial; sin embargo, una de las principales causas es el incumplimiento de las expectativas laborales. Autores como Boschma y Groen (2008), Ferri-Reed (2014), y Herbison y Boseman (2009), afirman que es responsabilidad de las empresas migrar a innovadores y más atractivos paquetes integrales (no solo salariales), dirigidos a satisfacer dichas expectativas.

Una de las teorías más conocidas, en camino a entender qué mueve a los trabajadores, es la de David McClelland, la cual ha sido utilizada para disminuir los índices de rotación y atraer a futuros colaboradores. En el libro titulado The achieving society (1961), el autor define los tres tipos de motivaciones compartidas por todas las personas, sin importar su origen, sexo o edad, que influyen en la toma de decisiones en cuanto a mantenerse o dejar sus puestos: necesidad de afiliación, necesidad de logro y necesidad de poder.

En este contexto, la presente investigación tiene como objetivo identificar qué esperan los centennials del mercado laboral, con base en la teoría de McClelland (1961), para considerar emplearse en una organización turística, así como los elementos de retención más atractivos, englobados en estrategias, planes y programas, que las empresas deberán priorizar y ofrecer en el corto plazo, a fin de convertirse en espacios atractivos que cubran las necesidades del perfil de interés.

Lo anterior lleva a las siguientes reflexiones: ¿cuáles son las principales motivaciones laborales de los estudiantes de turismo, próximos a insertarse en su primera experiencia profesional en una organización turística?, ¿cuáles son las variables que determinan su estabilidad en una empresa?, y ¿cómo se relacionan esas motivaciones con la teoría de David McClelland? 


\section{Antecedentes}

La literatura relacionada con las motivaciones, gustos, principios y expectativas de los centennials es tan nueva como limitada. A continuación, se expresan los hallazgos de algunos autores que han estudiado sus características, basados en diversos acontecimientos que forman parte de esta generación, así como de las precedentes.

Los Baby boomers (1946-1964), también llamados Alfa boomers o Golden boomers, son descendientes de la Segunda Guerra Mundial. Se caracterizan por ser pioneros de revoluciones sociales, en busca de un mundo de paz y armonía, rechazando los conflictos bélicos. Priorizan el respeto y la disciplina, son ahorradores y acumuladores, los que más leen, comen en casa y evitan caer en compras de impulso. Su motivación principal es la familia (Boschma y Groen, 2008; Hershatter y Epstein, 2010).

Los individuos de la Generación X (1965-1979), conocida igualmente como Peter Pan o Gen X, reciben su nombre de los Baby boomers, debido a la dificultad para entenderlos, pues no tenían rumbo o sentido. Crecieron en una época marcada por el escepticismo y la reforma social; se convirtieron en grandes impulsores de la tecnología, transformándose en seres consumistas que dejaron los aparatos electrónicos (fijos), para sustituirlos por los dispositivos portátiles (móviles). Son ambiciosos, buscan estudiar y escalar posiciones cuanto antes sea posible (Karriker y Hartman, 2018; Parry y Urwin, 2017).

La Generación Y (1980-2000) está compuesta por personas con una actitud desafiante y retadora, cuestionan todo. El internet ha sido parte de su vida y están inmersos en una sociedad con acceso a la información en todo momento. Jonsen et al. (2012), en su libro Cómo gestionar a los nativos digitales, comentan al respecto: "asumiendo que tienen derecho a ciertas cosas, creen que el mundo les debe algo, esto los convierte en personajes instantáneos que han desarrollado mentes con hipertexto prefiriendo el acceso sencillo, rápido y aleatorio de la información" (p. 62).

A la par, en su pensamiento se vuelven dictaminadores que pueden castigar o hacer populares a las organizaciones dependiendo de cómo se sienten tratados, por medio de las redes sociales (Bialik y Fry, 2019; Bizzi, 2017; Bosch et al., 2019; Espinoza y Ukleja, 2016; Fischer y Espejo, 2018; Karriker y Hartman, 2018; Holland et al., 2016; Murillo, 2017). 
La Generación Z, también denominada centennials, son nativos digitales, personas que nacieron y están creciendo con los avances tecnológicos, son impulsivos, lo que se refleja en un mayor empoderamiento. Están llenos de entretenimientos y se consideran hábiles en técnicas sociales de conexión tecnológica. La mayoría creció sin preocupaciones y confía en la prosperidad material e intelectual (Boschma y Groen, 2008).

Para Jonsen et al. (2012), los centennials han creado un perfil de personalidad basado más en la diversión que en las obligaciones y responsabilidades, son liberales en muchos sentidos, les gusta establecer sus propios horarios y reglas, evitan realizar tareas que no les satisfacen, y los retos y el reconocimiento en público les producen placer.

Asimismo, esta generación es la primera que ve a la tecnología como parte de su mundo. No tuvieron que adecuarse a los cambios técnicos y consideran a las actualizaciones algo normal de su vida (Carter y Walker, 2018). Tener dominio digital puede ser una ventaja, pues gracias a esta característica la incorporación al mundo laboral y la adaptación a las nuevas tecnologías les es natural (Ferri-Reed, 2014). También, aumentan su confianza para obtener ayuda, crear ideas o retroalimentación sincrónica, gracias al acceso a la información que en otras épocas no tendrían a su alcance (Jonsen et al., 2012).

Herbison y Boseman (2009) los describen como individuos que han estado rodeados por familiares, quienes les apoyan sobremanera en cualquier aspecto de sus vidas, alentándolos y motivándolos permanentemente en actividades extraacadémicas. Los estudios complementarios han logrado que tengan una habilidad superior en la solución de problemas y enfoque a resultados. Estos contextos de familia crean un sujeto que no se preocupa con facilidad, arraigado a casa de sus padres y creador de ambientes de respeto en sus espacios (Gibson et al., 2009).

Al hablar de organizaciones se entiende igualmente que se habla de personas, ambas se benefician unas de otras. Mientras que las primeras obtienen sus objetivos por medio de los individuos, las segundas logran sus metas a través de una estabilidad que se identifica como sinergia empresarial, donde los esfuerzos resultan en un ganar-ganar entre la organización y sus colaboradores. Cuando alguna de estas dos entidades obtiene más provecho sobre la otra, se pierde el equilibrio y surge la llamada rotación de personal. 
Como se indicó líneas arriba, dicha rotación es multivariable. Entre las distintas razones para que un empleado opte por abandonar una organización y contratarse en otra están: los cambios de entornos empresariales, la falta de comunicación o clarificación en metas y objetivos, la inadaptabilidad a normas y procedimientos, la diferencia del perfil de puestos versus el perfil del trabajador, o un ámbito competitivo con ofertas superiores (Boschma y Groen, 2008; Ferri-Reed, 2014; Golik, 2013; Haserot, 2009; Jonsen et al., 2012; Kowske et al., 2010; Meister, 2012; Thompson y Gregory, 2012).

En la actualidad, los jóvenes y su interés por encontrar un equilibrio entre la vida profesional y la personal son un tema abordado por varios investigadores (Bialik y Fry, 2019), por lo que muchas instancias ven este asunto como un elemento clave para reducir el índice de rotación, al afinar sus procesos en la gestión del capital humano y lograr con ello una mejor retención de los perfiles centennial.

En cuanto a la rotación, de acuerdo con las generaciones que convivan en una organización, deberán identificarse las variables principales que provocan este efecto, lo cual ha de entenderse como que el comportamiento humano, con relación a lo psicológico y lo tecnológico, hará que los procesos varíen, impactando en que se logre o no una retención de la fuerza de trabajo (Twenge, 2010).

Golik (2013) afirma que, a ojos de los centennials, el hecho de que los Baby boomers dediquen toda su existencia a una empresa, consumidos en lo personal y con sacrificios hacia sus familias, para ser despedidos luego de años de esfuerzo, es una situación que no están dispuestos a vivir (Solnet et al., 2012).

Tyler (2013) comenta que la Generación Z tiene una opinión puntual y directa respecto a la importancia de un empleo, que dista de las otras. El tema de la movilidad a corto o mediano plazo les es natural, inclusive satisfactoria; así, la decisión de retirarse de una empresa para buscar otras alternativas les produce un sentimiento de reto (Ertas, 2015; George y Wallio, 2017; Rani y Mukherjee, 2017).

Otras características de este perfil, que destacan Jonsen et al. (2012), son: a) quieren reconocimiento inmediato, por más mínimo que sea, b) desean que sus opiniones sean escuchadas, c) rechazan la lectura y presentan problemas de concentración, d) cuentan con una iniciativa mayor, así como capacidad para resolución de conflictos, e) se motivan por medio de retos y desafíos, y f) gustan de ser parte de un equipo, 
pero que las metas y logros se establezcan de manera abierta y consensuada (González, 2011).

En México, la media del índice de rotación de personal es de $16.75 \%$, el más alto de Latinoamérica (Meza, 2019). Dentro de la industria de servicios, especialmente en el sector restaurantero, puede llegar hasta el 50\% (Kelly Mercadotenia, 2017). Para Carter y Walker (2018), la atracción y la retención de recursos humanos son factores que adquieren mayor importancia ante la escasez de talento y afirman que es mejor y más barato conservar que reclutar nuevos candidatos.

Con lo anterior, se considera importante invertir lo que sea necesario en procesos adecuados de selección, complementados con un paquete atractivo de prestaciones para los trabajadores, quienes no resultaban tan críticos antes, pero ahora muestran cambios en la forma de valorar un puesto desde la perspectiva del significado y aporte que puede traer a sus vidas.

En ese tenor, los responsables de capital humano enfrentan grandes retos con las generaciones actuales, en particular por sus altas expectativas, la falta de arraigo para mantenerse por largo tiempo en un mismo departamento, la poca tolerancia, así como los deseos de superación inmediata, lo que juega un rol común en los centennials y provoca que su retención sea más complicada (Torrado, 2015).

Es en este punto donde los postulados de motivación son útiles para los profesionales del reclutamiento, como los siguientes:

a) Teoría de la jerarquía de necesidades, de Maslow (1943);

b) Teoría de la motivación e higiene, de Herzberg y Mausiner (1959);

c) Teorías X y Y, de McGregor (1985);

d) Modelo jerárquico ERC, de Alderfer (1969), en el que ERC son las siglas de existencia, relación y crecimiento;

e) Teoría de valencias y expectativas, de Vroom (1994), con las aportaciones de Porter y Lawler;

f) Teoría del establecimiento de metas, de Locke et al. (1990);

g) Teoría de la equidad, de Adams (1963; 1965); y

h) Teoría de las necesidades aprendidas, de McClelland (1961). 
Este último sustenta sus estudios en torno a las necesidades humanas que tienen los trabajadores, mediante la comparación y análisis de diversos colaboradores operativos y ejecutivos de distintas empresas. Concluye que existen tres grandes necesidades que destacan a la hora de motivarse para desempeñar una actividad laboral. Según McClelland estas se entienden como procesos inconscientes y se dividen en tres categorías:

a) Necesidad de afiliación. Personas que tienen fuertes deseos de ser parte de un grupo social, ávidas de gustar a los demás miembros, con un sentimiento de empatía. Prefieren la colaboración a la competición, no les agradan las situaciones riesgosas o de incertidumbre.

b) Necesidad de logro. Estos individuos buscan de manera intensa alcanzar metas, que involucren un nivel superior de desafío. Anhelan tomar riesgos controlados para lograr sus objetivos, y ansían retroalimentación sobre las actividades que desempeñan.

c) Necesidad de poder. Son aquellos que pretenden controlar a otros e influir en su comportamiento. Este punto se divide en dos: los del poder socializado (preocuparse más por los demás), y los del poder personal (la obtención de autoridad para su propio beneficio).

La teoría de las necesidades aprendidas de McClelland es el eje conductual de este documento, en el que se propone evaluar y diagnosticar las necesidades que expresan los centennials a la hora de elegir un proyecto laboral. La situación actual obliga a que las empresas propongan novedosos esquemas de empleo, que transformen los procesos y estructuras de gestión del capital humano a través de objetivos claros e innovadores, con la finalidad de poder reclutar y dinamizar a los próximos egresados de las universidades, para involucrarlos en una vida profesional sin que sacrifiquen sus expectativas personales.

Bussin y Brigman (2019) enlistan una serie de variables relevantes en las técnicas de atracción de personal en las organizaciones, dirigidas a perfiles centennials, así como la estrategia de retención de personal Employer Branding, conformada para satisfacer y promover la lealtad entre los empleados. Aquí pueden entrar en sinergia valores, comportamientos, al potenciar talentos de los presentes y futuros colaboradores en los negocios turísticos, con la satisfacción de sus necesidades de afiliación, logro y poder. 
El objetivo es lograr la identificación de las principales motivaciones laborales entre los jóvenes, hacia su primera experiencia profesional, lo cual implica establecer relaciones entre múltiples variables. Para ello, se contempla pertinente hacer uso del análisis factorial exploratorio, una técnica multivariante de aproximación estadística que permitirá revelar las interrelaciones de las variables de interés, a través de encontrar dimensiones (factores) subyacentes, comunes entre ellas. Es decir, dicho análisis será la manera de condensar la información recolectada y organizarla en un número más pequeño, sin perder datos valiosos (Hair et al., 1999).

En este método multivariable, se considera el supuesto de que las variables observadas, en lo individual, tienen una parte común al resto y otra parte que es específica. Es en esta última donde, además de la especificidad propia, se aprecian los errores de medida (Barbero, 2013). Lo que importa entonces es que la parte común pueda explicarse por una serie de factores, que son los que se calcularán como resultado de la investigación.

El análisis factorial exploratorio es útil debido a que se está en la búsqueda de una estructura entre una serie de variables cuantitativas, de ahí que se obtenga la información de los datos mismos, sin restricciones a priori sobre la estimación de los componentes o el número de ellos a ser extraído. En resumen, no hay preconcepciones sobre la estructura de los datos.

Además, se establece bajo esta técnica que los factores (latentes) pueden estar correlacionados o bien ser independientes. Más de un factor podría afectar a las variables, pero los errores no se correlacionan entre sí. Dichas variables están afectadas por un único término de error y los factores o las variables latentes no se correlacionan con los términos de error (Barbero, 2013).

\section{Metodología}

El objetivo se centró en identificar las expectativas laborales que tienen los próximos egresados de la carrera de turismo, como parte de la Generación Z, dispuestos a insertarse a corto plazo en empresas de servicios turísticos. La perspectiva teórica aplicada fue la de las necesidades aprendidas de McClelland (1961), que orienta hacia las motivaciones para emplearse en una organización; asimismo, se observaron los elementos de retención más atractivos que las compañías tienen por ofrecer. 
Ante ello, se diseñó una investigación no experimental de alcance correlacional, en la que se estableció la relación entre variables (U.S. Department of Education, 2005) que se hipotetiza conforman las perspectivas que poseen los futuros colaboradores (de perfil centennial) para unirse o permanecer en una organización, con el fin de valorar su importancia (o prioridad) entre la población estudiada.

Robbins (1999) y Schultz (1992) describen como factor de rotación la insatisfacción del trabajador, definida como la discrepancia entre la cantidad de recompensas que espera de las que, efectivamente, recibe. Ello se comprende como que un empleado debe estar satisfecho (con recompensas justas y equitativas) para tener una estabilidad, y que la mayoría de los individuos descontentos son jóvenes solteros, quienes cuentan con mayor libertad de abandonar sus puestos que los colaboradores de más de 50 años, ello invita a plantear la primera hipótesis:

H1: la combinación de actividades laborales y personales impacta de manera directa en el índice de rotación de personal.

Cabe señalar que en ocasiones dichas actividades encuentran un balance. Al respecto, Kahn (2017) estipula que los individuos en toda organización juegan roles distintos, conviviendo y desarrollándose en sus puestos en tres niveles: el físico, el cognitivo y el emocional. En cuanto a este último, existen estudios que profundizan en el concepto de salario emocional, como las de Espinoza y Toscano (2020), quienes afirman que a los colaboradores no solo los mueve la remuneración económica, sino que buscan más allá, teniendo intereses heterogéneos de satisfacción personal, como el de integrar un equipo que sea percibido como sólido y dinámico.

Es por ello, de acuerdo con Cloninger (2002), que la pertenencia a un colectivo, ya sea pequeño o grande, ayuda a aportar sentido a lo que se hace en el día a día; además, el contacto personal y las relaciones sociales favorecen estos lazos de pertenencia, por lo que, aunado al fundamento anterior, se plantea la siguiente hipótesis:

H2: la necesidad de afiliación laboral es un factor determinante para que personas con perfil centennial se mantengan en una organización.

Este estudio transversal se define en un momento en el tiempo (Ato et al., 2013). El levantamiento de datos se realizó entre febrero y marzo del año 2020. El marco de población estuvo compuesto por los estudiantes de la Licenciatura de Turismo Sustentable y Gestión Hotelera de la Universidad del Caribe, ubicada en Cancún, 
Quintana Roo, próximos a egresar en el periodo de otoño e invierno del 2020, nacidos entre los años 1994 y 1999.

La representatividad de la población elegida radicó no solo en que cumplen con fechas de nacimiento previas al 2005, según Magallón (2016), sino en su actitud desafiante y retadora, la conectividad de la que gozan en esta época digital, el empoderamiento que perciben en los distintos ámbitos de sus vidas, las motivaciones y expectativas laborales que mantienen en el corto plazo, los diversos estratos sociales a los que pertenecen, y sus dinámicas de núcleos familiares múltiples.

Fue posible realizar un censo a través de la plataforma Google Forms, utilizando el correo institucional de dicha población. Este proceso virtual permitió crear una liga vinculada al cuestionario, que sería alimentado por los sujetos bajo análisis. El llenado individual de este instrumento no implicó más de 10 minutos. La tasa de respuesta obtenida fue de 9 de cada 10 inscritos en esta cohorte, pues 10 de los estudiantes (contactados en la última semana de marzo, cuando inició la contingencia por la pandemia del COVID-19), no respondieron al formulario.

Las razones por las que no participaron fueron variadas: a) no abrieron el correo con la invitación a la encuesta en línea, b) tuvieron nulo o limitado acceso a internet, c) regresaron a sus lugares de origen (fuera de Cancún), y d) experimentaron condiciones que dificultaron la conectividad. Así, la tasa de no respuesta fue de 1:10.

Las variables seleccionadas en el instrumento fueron tomadas parcialmente de la propuesta de Madero y De la Garza (2017) y de Madero et al. (2016), englobados en los planteamientos de McClelland.

Tabla 1. Variables de expectativas laborales

\begin{tabular}{|l|l|l|l|l|}
\hline 1. Liderazgo & 2. Ubicación & 3. Alimentos & 4. Infraestructura & 5. Bienestar \\
\hline 6. Salud & 7. Balance & 8. Desarrollo & 9. Transporte & 10. Clima laboral \\
\hline 11. Sustentabilidad & 12. Certificaciones & 13. Salario & 14. Procedimientos & 15. Movilidad \\
\hline
\end{tabular}

Fuente: Elaboración propia.

El instrumento consistió en un cuestionario formado en su mayoría por preguntas cerradas y algunas abiertas, para recolectar los valores de las 15 variables (tabla 1), y 12 de perfil demográfico (año de nacimiento, género, situación de empleo, conocimientos informáticos, idiomas) y psicográfico (motivación, habilidades, au- 
topercepción, principales distracciones, cuidados personales, gustos personales e intereses).

Dichas variables se detallan a continuación, de acuerdo con las categorías de necesidades aprendidas que propone McClelland (1961).

Necesidades de afiliación:

1) Liderazgo: que el supervisor o jefe inmediato sea considerado un líder.

2) Ubicación: que la localización de la empresa donde se desarrollan las actividades laborales sea importante para elegirla.

3) Alimentos: contar con un comedor y alimentos saludables e higiénicos.

4) Infraestructura: acceso a materiales, equipos, herramientas, espacios y suministros a fin de desarrollar las funciones del puesto.

5) Bienestar: tener instalaciones de descanso, entretenimiento y ejercicio para colaboradores.

6) Salud: ofrecer servicios de salud y bienestar integral.

Necesidades de logro:

7) Balance: que las actividades laborales permitan tener un equilibrio entre la vida personal y la vida profesional.

8) Desarrollo: que la empresa presente un plan de crecimiento, capacitación y adiestramiento.

9) Transporte: contar con transporte de personal para traslados.

10) Clima laboral: promover un ambiente positivo.

11) Sustentabilidad: que se implementen (o estén en planeación) programas de sustentabilidad y manejo adecuado de recursos.

12) Certificaciones: impulsar reconocimientos, certificaciones o galardones.

Necesidades de poder:

13) Salario: contar con un ingreso adecuado.

14) Procedimientos: que la empresa opere con reglamentos, manuales y procedimientos establecidos.

15) Movilidad: que se ofrezcan programas de movilidad nacional o internacional. 
Para las respuestas se utilizó una escala Likert de medición del 1 al 5, siendo totalmente en desacuerdo (1), en desacuerdo (2), neutro (3), de acuerdo (4), y totalmente de acuerdo (5). Para el bloque del perfil se combinaron escalas de medición nominales y ordinales.

El instrumento presentó una consistencia interna válida, ya que el coeficiente Alfa de Cronbach resultó en 0.86 . En la evaluación de la confiabilidad se aplicó este método, por ser el más utilizado para la estimación de este dato, mismo que permite señalar la magnitud de la covarianza de los ítems que se incluyen en el instrumento, y la medida en la que el constructo (o tema de estudio) está presente en ellos (Ventura-León y Caycho-Rodríguez, 2017).

En este análisis factorial tipo $\mathrm{R}$ (el diseño más común dentro de esta técnica), se siguió un método de cinco pasos, que explican Hair et al. (1999), con la serie de variables de investigación para identificar sus dimensiones latentes:

Paso 1. Diseño del análisis factorial, en el que se calculó una matriz de correlación. Se delineó en términos del número de variables (15, seleccionadas para explicar el supuesto de contratación), sus propiedades de medición y los tipos, así como el tamaño de muestra adecuado, pues en el censo que se realizó se eligieron a conveniencia 100 casos, lo que permitió respetar la ratio aceptable de 10 a uno, con relación a las variables finales que se procesaron; el criterio para conservar una de ellas con significancia estadística, fue que su coeficiente de correlación ( $r$ ) fuera mayor a $|0.5|$. El nivel de significancia (a) utilizado en las pruebas de hipótesis fue de 0.05 .

Paso 2. Revisión de los supuestos del análisis factorial, que tienen que ver con la distribución de normalidad, homocedasticidad y linealidad.

Paso 3. Estimación de los factores y valoración del ajuste, para el que se utilizó el análisis de componentes principales, que permitiría ponderar la varianza total y la referida estimación de los factores, los cuales presentan proporciones bajas de varianza única. Asimismo, el interés en este estudio fue la predicción y el obtener una única solución.

Paso 4. Interpretación de los factores, que inició con:

a) El cálculo de la matriz de factores no rotados, lo cual permitió conocer de manera preliminar cuántos factores extraer para el modelo. Esta matriz despliega 
las cargas de cada variable por factor; además, con ella es posible visualizar la mejor combinación lineal y las subsecuentes;

b) Rotación de factores con el método ortogonal de aproximación Varimax, que maximiza la suma de varianzas de las cargas de la matriz de factores; por tanto, lo conveniente es que estas sean cercanas a $-10+1$, pues eso indicaría que hay una clara asociación negativa o positiva, respectivamente, entre la variable y el factor;

c) Valoración de la significación de cargas factoriales, asegurando la significación práctica. Esto se resumió en que cualquier factor con carga de 0.55 o más (en términos absolutos) sería por demás significativo, pues la cantidad de casos lo permite. Lo anterior posibilitó expresar la carga como el porcentaje de varianza explicada debida al factor, elevando al cuadrado y multiplicando por 100;

d) Valoración de la comunalidad, que representa la proporción de varianza con la que contribuye cada variable a la solución final. Así pues, si la comunalidad es menor a 0.5 se considera que esa variable está carente de explicación suficiente; $y$

e) Etiquetación de los factores, que está muy relacionada con el conocimiento especializado del material por parte del investigador, pues se fabricaron intuitivamente, a partir de las asociaciones y cargas obtenidas.

Paso 5. Validación del análisis factorial, con mecanismos como el de la división de grupos, para evaluar el grado para generar nuevos resultados.

En la examinación de los datos y la elaboración de recursos visuales (tablas y gráficos) se utilizó GNU PSPP v.1.2.0 y Excel v.365. Una vez hecha la validación del modelo, se concluiría sobre las hipótesis planteadas.

\section{Resultados}

Este apartado se organizó en tres partes:

1) Análisis descriptivo de las variables demográficas y psicográficas, para identificar la composición de la población centennial, entre los estudiantes de la Licenciatura de Turismo Sustentable y Gestión Hotelera de la Universidad del 
Caribe, quienes se perfilan a egresar e insertarse en empresas de vocación turística.

2) Análisis descriptivo ponderado, que permite ubicar las variables de mayor relevancia para contratación entre los sujetos de estudio.

3) Análisis factorial exploratorio, dirigido a validar las correlaciones entre variables y determinar los factores de constructos.

\section{Análisis demográfico y psicográfico}

En la tabla 2 se muestra la información relacionada con los 100 sujetos que contestaron el instrumento, en cuanto a género, situación de empleo, nivel de conocimientos informáticos y dominio de idiomas extranjeros (cantidad). Predomina el género femenino. Por lo menos 80 de ellos han tenido experiencia de trabajo formal, la mayoría domina la paquetería Office y otro software que se utiliza en organizaciones de servicios turísticos y más de 90 estudiantes hablan inglés.

Tabla 2. Características demográficas de los sujetos de estudio

\begin{tabular}{|l|c|c|}
\hline \multicolumn{1}{|c|}{ Variable $y$ amplitud } & Número & Porcentaje \\
\hline 1. Sexo & & \\
\hline Masculino & 37 & $37 \%$ \\
\hline Femenino & 63 & $63 \%$ \\
\hline 2. Situación laboral & & \\
\hline Se encuentra laborando & 23 & $23 \%$ \\
\hline No está laborando, pero ha trabajado & 60 & $60 \%$ \\
\hline Nunca ha trabajado & 6 & $6 \%$ \\
\hline Solo ha realizado prácticas & 11 & $11 \%$ \\
\hline 3. Conocimientos informáticos & & \\
\hline Maneja solo Office & 40 & $40 \%$ \\
\hline Domina Office y otro software turístico & 60 & $60 \%$ \\
\hline 4. Nivel de idiomas & & $82 \%$ \\
\hline Solo habla español & 0 & $0 \%$ \\
\hline Habla español e inglés & & \\
\hline Habla más de dos idiomas & & \\
\hline
\end{tabular}

Fuente: Elaboración propia. 
En la tabla 3 se observan las principales motivaciones, habilidades, descripción propia y sus principales intereses. Algunos sujetos son motivados por la familia, reconocen que tienen habilidades de socialización, de ahí que se describan a sí mismos como amistosos, y a la tercera parte de ellos le gustaría viajar.

Tabla 3. Principales motivaciones de los sujetos de estudio

\begin{tabular}{|c|c|c|}
\hline Variable y amplitud & Número & Porcentaje \\
\hline \multicolumn{3}{|l|}{ 5. Motivación principal } \\
\hline Familia & 40 & $40 \%$ \\
\hline Superación personal & 19 & $19 \%$ \\
\hline Tener un mejor empleo & 13 & $13 \%$ \\
\hline Tener una vida estable & 9 & $9 \%$ \\
\hline Otros & 19 & $19 \%$ \\
\hline \multicolumn{3}{|l|}{ 6. Habilidades } \\
\hline Socialización & 38 & $38 \%$ \\
\hline Aprendizaje & 25 & $25 \%$ \\
\hline Creatividad & 18 & $18 \%$ \\
\hline Trabajo en equipo & 5 & $5 \%$ \\
\hline Otras & 14 & $14 \%$ \\
\hline \multicolumn{3}{|l|}{ 7. Descripción de ellos mismos } \\
\hline Amistoso & 23 & $23 \%$ \\
\hline Inteligente & 16 & $16 \%$ \\
\hline Honesto & 11 & $11 \%$ \\
\hline Proactivo & 10 & $10 \%$ \\
\hline Responsable & 9 & $9 \%$ \\
\hline Otras & 31 & $31 \%$ \\
\hline \multicolumn{3}{|l|}{ 8. Intereses } \\
\hline Viajar & 33 & $33 \%$ \\
\hline Conocer gente & 24 & $24 \%$ \\
\hline Estudiar más & 14 & $14 \%$ \\
\hline Apoyar a otros & 5 & $5 \%$ \\
\hline Otros & 24 & $24 \%$ \\
\hline
\end{tabular}

Fuente: Elaboración propia.

La tabla 4 muestra las principales distracciones, cuidados personales, gustos e intereses que tienen en la vida. Se destaca el gusto por la lectura y el arte (cerca de la cuarta parte) y la buena alimentación, en más del 50\%. 
Tabla 4. Entretenimiento, cuidado y gustos de los sujetos de estudio

\begin{tabular}{|c|c|c|}
\hline Variable y amplitud & Número & Porcentaje \\
\hline \multicolumn{3}{|l|}{ 9. Principales distracciones } \\
\hline Lectura & 26 & $26 \%$ \\
\hline Música & 17 & $17 \%$ \\
\hline Series & 14 & $14 \%$ \\
\hline Videojuegos & 12 & $12 \%$ \\
\hline Otros & 31 & $31 \%$ \\
\hline \multicolumn{3}{|l|}{ 10. Cuidados personales } \\
\hline Buena alimentación & 52 & $52 \%$ \\
\hline Ejercicio/Deporte & 31 & $31 \%$ \\
\hline Higiene & 9 & $9 \%$ \\
\hline Otras & 8 & $8 \%$ \\
\hline \multicolumn{3}{|l|}{ 11. Gustos personales } \\
\hline Arte & 23 & $23 \%$ \\
\hline Comida & 19 & $19 \%$ \\
\hline Naturaleza & 15 & $15 \%$ \\
\hline Otros & 43 & $43 \%$ \\
\hline \multicolumn{3}{|l|}{ 12. Intereses en la vida } \\
\hline Viajar & 36 & $36 \%$ \\
\hline Buen empleo & 25 & $25 \%$ \\
\hline Aprender & 19 & $19 \%$ \\
\hline Otros & 20 & $20 \%$ \\
\hline
\end{tabular}

Fuente: Elaboración propia.

\section{Análisis descriptivo ponderado}

La encuesta permitió recolectar información para el procesamiento y análisis descriptivo, planteando una relación entre las variables de expectativas laborales, con la finalidad de identificar cuáles son las que más influyen al momento de tomar la decisión de ingresar a una empresa o no, así como seguir en ella o dejarla para buscar un nuevo espacio para laborar.

Después de obtener los estadísticos descriptivos sobre la dispersión (tabla 5), se pudo apreciar que tanto ubicación (V5), salario (V4), transporte (V8), así como bienestar (V14), son las que mayor variabilidad registraron (SD > 0.75), con respecto al resto. 
Tabla 5. Estadísticos de dispersión de las variables de expectativas laborales

\begin{tabular}{|c|c|c|c|c|c|}
\hline \multicolumn{2}{|c|}{ Variable } & $\begin{array}{l}\text { Media } \\
\text { (M) }\end{array}$ & $\begin{array}{l}\text { Err. Est. Media } \\
\text { (EEM) }\end{array}$ & $\begin{array}{l}\text { Desv. Estd. } \\
\text { (SD) }\end{array}$ & $\begin{array}{l}\text { Coef. Variación } \\
\text { (CV) }\end{array}$ \\
\hline Balance & (V1) & 4.72 & 0.05 & 0.51 & $10.89 \%$ \\
\hline Desarrollo & (V2) & 4.72 & 0.06 & 0.55 & $11.69 \%$ \\
\hline Liderazgo & (V3) & 4.66 & 0.06 & 0.61 & $13.02 \%$ \\
\hline Salario & (V4) & 4.51 & 0.08 & 0.77 & $17.12 \%$ \\
\hline Ubicación & (V5) & 4.30 & 0.08 & 0.82 & $19.13 \%$ \\
\hline Alimentos & (V6) & 4.68 & 0.06 & 0.65 & $13.88 \%$ \\
\hline Infraestructura & (V7) & 4.81 & 0.04 & 0.44 & $9.20 \%$ \\
\hline Transporte & (V8) & 4.50 & 0.08 & 0.76 & $16.86 \%$ \\
\hline Clima laboral & (V9) & 4.81 & 0.05 & 0.51 & $10.53 \%$ \\
\hline Procedimientos & (V10) & 4.59 & 0.07 & 0.70 & $15.20 \%$ \\
\hline Certificaciones & (V11) & 4.46 & 0.07 & 0.70 & $15.75 \%$ \\
\hline Sustentabilidad & (V12) & 4.77 & 0.05 & 0.49 & $10.26 \%$ \\
\hline Movilidad & (V13) & 4.57 & 0.07 & 0.67 & $14.67 \%$ \\
\hline Bienestar & (V14) & 4.39 & 0.08 & 0.76 & $17.40 \%$ \\
\hline Salud & (V15) & 4.78 & 0.05 & 0.52 & $10.96 \%$ \\
\hline
\end{tabular}

Nota: Casos válidos $=100$; casos con valor(es) perdido(s) $=0$.

Fuente: Elaboración propia.

En la figura 1 se visualizan las variables que los jóvenes consideran prioritarias:

1) Contar con infraestructura, herramientas y equipos aptos para desarrollar las funciones - $V 7$ - $(M=4.81 ; S D=0.44)$;

2) Tener un balance de vida personal y profesional -V1- $(M=4.72 ; S D=0.51)$;

3) Contar con un clima laboral positivo -V9- $(\mathrm{M}=4.81$; $\mathrm{SD}=0.51)$;

4) Implementar programas de sustentabilidad y de manejo adecuado de recursos $-V 12-(M=4.77 ; S D=0.49)$;

5) Ser parte de un plan de crecimiento, capacitación y adiestramiento -V2- (M $=4.72 ; \mathrm{SD}=0.55) ; \mathrm{y}$

6) Tener servicios de salud y bienestar integral para colaboradores -V14- ( $M=$ 4.39; DS = 0.79).

En el semáforo que se muestra abajo de la figura 1, se enlista la totalidad de las variables de expectativas laborales. De acuerdo con la intensidad del color, el verde fue el mejor ponderado (se sumaron los puntos 4 y 5 , que expresaban acuerdo con 
el ítem descrito), y el rojo el menor calificado. Destaca que 23 de los 100 encuestados dijeron no estar interesados en la localización de la empresa que seleccionarán para trabajar, pues evaluaron ese punto como neutral (3 puntos en escala de Likert).

Figura 1. Ponderación de variables de expectativas laborales

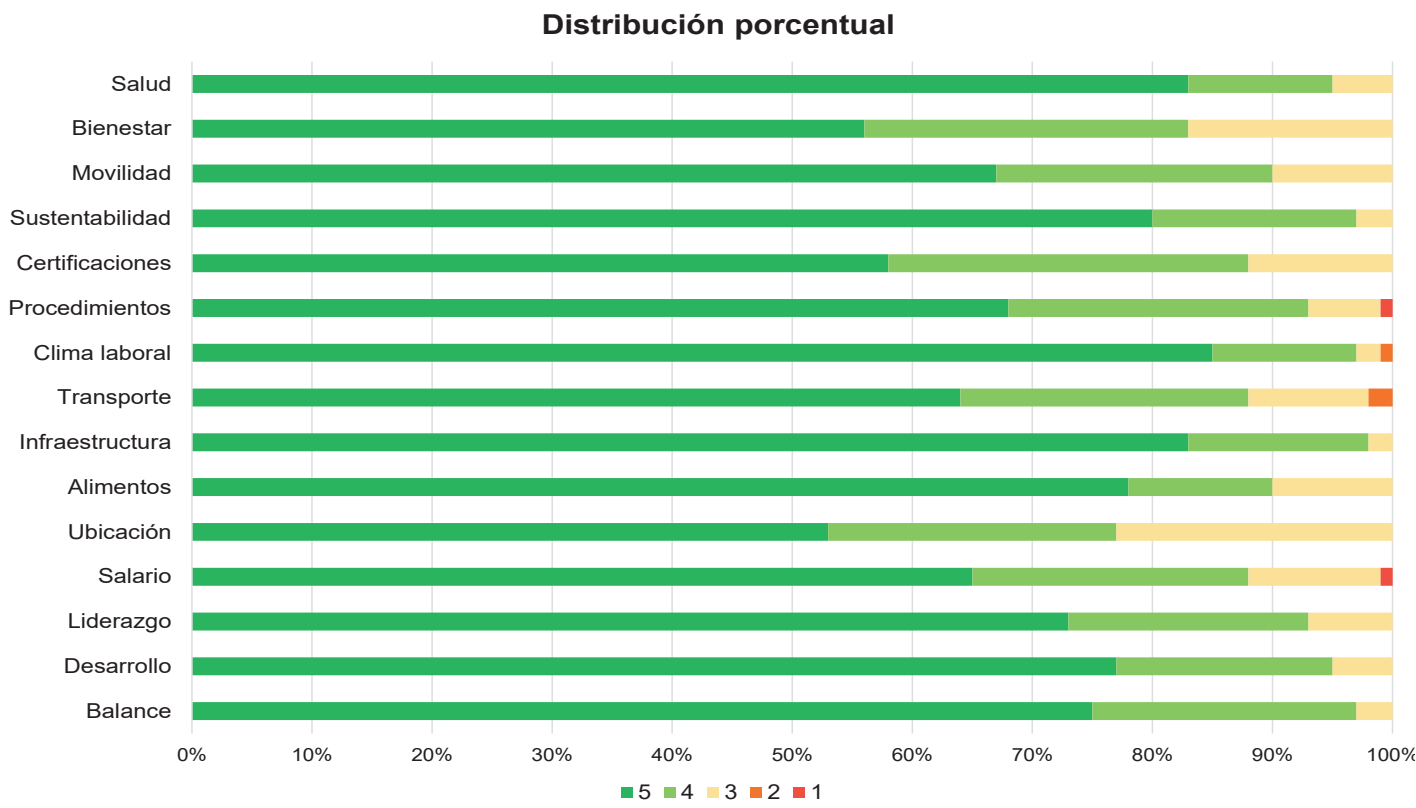

\begin{tabular}{l|c}
\multicolumn{1}{c|}{ Variable } & $\begin{array}{c}\text { \% positivo } \\
\text { (pesos de 4 y 5) }\end{array}$ \\
\hline Infraestructura & 98 \\
\hline Balance & 97 \\
\hline Clima laboral & 97 \\
\hline Sustentabilidad & 97 \\
\hline Desarrollo & 95 \\
\hline Salud & 95 \\
\hline Liderazgo & 93 \\
\hline Procedimientos & 93 \\
\hline Alimentos & 90 \\
\hline Movilidad & 90 \\
\hline Salario & 88 \\
\hline Transporte & 88 \\
\hline Certificaciones & 88 \\
\hline Bienestar & 83 \\
\hline Ubicación & 77
\end{tabular}

Fuente: Elaboración propia. 


\section{Análisis factorial exploratorio}

Junto con el análisis descriptivo, se asociaron las variables para la obtención del coeficiente de correlación ( $r$ ). La tabla 6 solo muestra aquellas en las que se registró un $r \geq|0.5|$ que fue significativo. Así, vemos que son 10 asociaciones lineales bivariadas las que aparecen como aceptables en el análisis factorial.

Tabla 6. Coeficiente de correlación

\begin{tabular}{|c|c|c|c|c|c|c|c|c|}
\hline Variable & Liderazgo & Alimentos & $\begin{array}{l}\text { Infra- } \\
\text { estructura }\end{array}$ & $\begin{array}{l}\text { Clima } \\
\text { laboral }\end{array}$ & $\begin{array}{l}\text { Proce- } \\
\text { dimientos }\end{array}$ & $\begin{array}{l}\text { Susten- } \\
\text { tabilidad }\end{array}$ & Bienestar & Salud \\
\hline Liderazgo & 1 & & & & & & & \\
\hline Alimentos & $0.34^{*}$ & 1 & & & & & & \\
\hline Infraestructura & $0.47^{*}$ & $0.63^{*}$ & 1 & & & & & \\
\hline Clima laboral & $0.38^{*}$ & $0.37^{*}$ & $0.38^{*}$ & 1 & & & & \\
\hline Procedimientos & $0.38^{*}$ & $0.55^{*}$ & $0.50^{*}$ & $0.43^{*}$ & 1 & & & \\
\hline Sustentabilidad & $0.31^{*}$ & $0.47^{*}$ & $0.45^{*}$ & $0.56^{*}$ & $0.52^{*}$ & 1 & & \\
\hline Bienestar & $0.35^{*}$ & 0.19 & $0.31^{*}$ & 0.19 & $0.32^{*}$ & $0.38^{*}$ & 1 & \\
\hline Salud & $0.53^{*}$ & $0.47^{*}$ & $0.65^{*}$ & $0.45^{*}$ & $0.52^{*}$ & $0.51^{*}$ & $0.52^{*}$ & 1 \\
\hline
\end{tabular}

Para este análisis se establecieron dos factores: F1 y F2. El primero, compuesto por variables de los tres tipos de necesidades (afiliación, logro y poder), y el segundo, integrado solamente por las que se enfocan en necesidades de afiliación. Después de la rotación ortogonal, los resultados se muestran en la tabla 7. 
Tabla 7. Matriz de factores del análisis de componentes Varimax

\begin{tabular}{|r|l|c|c|c|}
\hline \multicolumn{1}{|c|}{ Variable } & F1 & F2 & Comunalidades \\
\hline V3- & Liderazgo & 0.37 & 0.61 & 0.51 \\
\hline V6- & Alimentos & 0.82 & 0.08 & 0.67 \\
\hline V7- & Infraestructura & 0.70 & 0.37 & 0.62 \\
\hline V9- & Clima laboral & 0.73 & 0.27 & 0.60 \\
\hline V10- & Procedimientos & 0.69 & 0.16 & 0.50 \\
\hline V12- & Sustentabilidad & 0.69 & 0.31 & 0.56 \\
\hline V14- & Bienestar & 0.05 & 0.90 & 0.81 \\
\hline V15- & Salud & 0.54 & 0.66 & 0.74 \\
\hline & Suma de cuadrados & & & Total \\
\hline & Autovalor & 3.08 & 1.96 & 5.03 \\
\hline & \% de traza* & Fuente: Elaboración propia. & & 62.93 \\
\hline
\end{tabular}

Por tanto, el F1 se compondrá de las variables alimentos (V6), infraestructura (V7), clima laboral (V9), procedimientos (V10) y sustentabilidad (V12); y el F2, por liderazgo (V3), bienestar (V14) y salud (V15). Con ello, al F1 se le puede denominar bloque $A$, y al F2 bloque $B$.

Asimismo, el modelo multivariante factorial exploratorio presentó una varianza explicada de casi $63 \%$ (porcentaje de traza), y la carga de cada factor solo se diferenció por 14 puntos porcentuales, en discrepancia con lo que se había registrado antes de la rotación (39 puntos, donde el F1 tenía una carga de 51\%). El Alfa de Cronbach para el instrumento con las variables de F1 resultó en 0.82, y el F2 en 0.70, ambos aceptables.

Hacia la validación del modelo, se fraccionó la base de datos con la mitad de los casos $(n / 2)$ componiendo la muestra de división 1 (D1) del registro 1 al 50, y la muestra de división 2 (D2) del 51 al 100. Los resultados se despliegan en la tabla 8. Al observar ambas, se percibe que las cargas entre factores se mantuvieron casi en la misma proporción, así como su varianza explicada; no sucedió así con variables como liderazgo (V3), bienestar (V14) y salud (V15), cuyos valores en la D1 fueron de $0.34,0.04$ y 0.56 , respectivamente, y en la $D 2$, se aprecian igual a $0.52,0.56$ y 0.87 , esto es que marcan más carga hacia el F1 en la muestra D2, que en el F1 de la D1. 
Clima laboral (V9) y sustentabilidad (V12) son las únicas variables que se cargan al F2, de la D1 a la D2. Así, también las comunalidades varían en dos de las variables (V3 y V14). Liderazgo, por su parte, descendió de 0.56 a 0.31 , y bienestar, de 0.80 a 0.35 . Tales cambios son grandes. Las tres puntuaciones no son convenientes para obtener un resultado concluyente.

Tabla 8. Validación del análisis de componentes por estimación de división de los casos Varimax

\begin{tabular}{|c|c|c|c|c|}
\hline & Variable & F1 & F2 & Comunalidades \\
\hline Muest & ra de división 1 ( & & & \\
\hline V3- & Liderazgo & 0.34 & 0.67 & 0.56 \\
\hline V6- & Alimentos & 0.84 & 0.1 & 0.72 \\
\hline V7- & Infraestructura & 0.65 & 0.52 & 0.70 \\
\hline V9- & Clima laboral & 0.69 & 0.43 & 0.65 \\
\hline V10- & Procedimientos & 0.82 & 0.15 & 0.69 \\
\hline V12- & Sustentabilidad & 0.64 & 0.38 & 0.56 \\
\hline V14- & Bienestar & 0.04 & 0.89 & 0.80 \\
\hline V15- & Salud & 0.56 & 0.68 & 0.77 \\
\hline Muest & ra de división 2 ( & & & \\
\hline V3- & Liderazgo & 0.52 & 0.21 & 0.31 \\
\hline V6- & Alimentos & 0.62 & 0.46 & 0.6 \\
\hline V7- & Infraestructura & 0.71 & 0.27 & 0.57 \\
\hline V9- & Clima laboral & -0.14 & 0.89 & 0.8 \\
\hline V10- & Procedimientos & 0.75 & 0.23 & 0.61 \\
\hline V12- & Sustentabilidad & 0.43 & 0.69 & 0.66 \\
\hline V14- & Bienestar & 0.56 & -0.19 & 0.35 \\
\hline V15- & Salud & 0.87 & -0.05 & 0.76 \\
\hline
\end{tabular}

Nota: Los cuadros resaltados se han dejado como referencia de las variables del modelo original, mostrado en la tabla 7. Fuente: Elaboración propia.

\section{Discusión}

En congruencia con el objetivo de esta investigación, se establecieron dos hipótesis cuyo cuestionamiento tuvo el siguiente resultado:

La hipótesis 1 es real. Relacionada con la premisa de que el balance entre las actividades laborales y las personales impacta de manera directa en el índice de rotación de personal, se observa que el equilibrio entre estas dos dimensiones es lo que más importa a los futuros trabajadores turísticos. 
Esta hipótesis no se refuta pues la variable balance (V1) fue la segunda más ponderada con $97 \%$ de peso, es decir, en el análisis descriptivo en el cual se utilizó la escala de Likert, los mayores valores (entre 5 y 4) fueron otorgados a la pregunta que representaba esta variable. Además, la $V 1$ resultó consistente en las respuestas de los sujetos bajo estudio, con un CV cercano al $10 \%$.

La hipótesis 2 fue rechazada. Afirmaba que la necesidad de afiliación laboral es un factor de retención para personas con perfil centennial; no obstante, con el análisis factorial exploratorio, las variables liderazgo (V3) y bienestar (V14) no permitieron la reducción de los factores considerados (F1 y F2), pues ambas presentan un cambio evidente al momento de realizar la validación del modelo. Es decir, para evitar el rechazo de la hipótesis, lo ideal sería que V3 y V14 no mostraran diferencias en sus comunalidades en el análisis de componentes, por estimación de división de los casos (D1 y D2).

En particular, en el caso de la hipótesis 2 se esperaba que se cumpliera a cabalidad la afirmación de que las necesidades de afiliación laboral, según la teoría de McClelland (1961), serían en conjunto los motivadores principales de retención. En el esfuerzo de buscar una reducción de variables a través del análisis factorial exploratorio, y de acuerdo con sus comportamientos específicos y de interdependencia, se pensaba que las variables de liderazgo (V3), ubicación (V5), alimentos (V6), infraestructura (V7), bienestar (V14) y/o salud (V15), todas o algunas de ellas conformarían un factor (F1 o F2), lo cual no sucedió.

Ante los resultados exploratorios derivados de la evidencia empírica, se contempla necesario conseguir una muestra adicional para validar dicha hipótesis; sin embargo, en el año en curso no será viable la obtención de dicha muestra, pues se requiere esperar a que la siguiente cohorte de estudiantes próximos a egresar, en el mismo programa educativo, esté cursando el periodo primavera 2021, con el fin de replicar las condiciones de la población referida.

Destaca aquí una de las variables que no se esperaba fuera atractiva para el perfil centennial: la de infraestructura (V7), siendo la más ponderada de las 15, con 98 puntos, al evidenciar que sí es de interés de los jóvenes contar con materiales, herramientas, equipos, espacios y suministros aptos para desarrollar sus funciones. Por el contrario, la variable salario (V4) (con 88), que pudiera pensarse sería la más atractiva, obtuvo 10 puntos menos que la más alta (V7). 
En resumen, el salario (V4) no es una prioridad entre los encuestados, por lo que no se recomienda que se le relacione con la rotación de personal en una próxima investigación. No obstante, sí se sugiere abrir una línea que valore elementos tales como materiales, herramientas, equipos, espacios y suministros para desarrollar tareas, a fin de determinar su influencia en la retención de los recursos humanos.

En un acto de reflexión práctica, el tema de expectativas laborales se ha abordado desde diversas posturas; sin embargo, sigue sin encontrarse la clave para la disminución de la rotación de personal, al ser una cuestión multifactorial (Boschma y Groen, 2008; Ferri-Reed, 2014). Conscientes de ello, el análisis aquí desarrollado es una exploración más, que pretende contribuir en esta área de oportunidad.

Los indicadores de rotación de personal, evaluaciones de desempeño y el alcance de los objetivos y metas establecidas, son aspectos que los gerentes generales, y en sí todo el comité ejecutivo de las empresas turísticas, deben fortalecer (Smith, 2010).

Proporcionar herramientas, equipos y espacios aptos para el desarrollo de tareas, permitir tener un equilibrio entre la vida personal y profesional, promover un clima laboral positivo, e implementar programas de sustentabilidad y manejo adecuado de recursos, con fines de potenciar a sus colaboradores, será la diferencia entre ser una empresa en peligro de cierre, o ser competitiva y atractiva para clientes internos y externos.

\section{Conclusiones}

Este trabajo se enfocó en identificar las expectativas laborales que tiene la Generación Z, según la teoría de McClelland (1961), con el propósito de entender mejor el fenómeno y las implicaciones de contratación en empresas turísticas, así como plantear esquemas de retención mayor, a través de estrategias, planes y programas de gestión de capital humano, para ser más atractivas a los ojos de las nuevas generaciones, como lo sugieren Thompson y Gregory (2012).

El análisis realizado permitió reconocer los intereses que priorizan los próximos egresados de turismo de la Universidad del Caribe (trabajadores potenciales en Cancún), con perfil de centennials, que son las necesidades de logro (balance, desarrollo, clima laboral y sustentabilidad) y de afiliación (infraestructura, bienestar y salud), antes de las de poder (procedimientos, salario y movilidad). 
Es indudable que la investigación puede tener un alcance más amplio, contemplando poblaciones universitarias que estudian turismo en otras Instituciones Educativas de nivel Superior (IES) en Cancún, pues todas atienden la oferta laboral de dicho destino turístico.

Con lo anterior, se considera pertinente suscitar una revisión de la oferta y demanda de relaciones contractuales, con un enfoque en las características demográficas de los centennials, que se adecúe al panorama turístico en curso. Esto permitirá, de acuerdo con Paradinas (2020), diseñar acercamientos en el ámbito que aborden la gestión del conflicto, para identificarlo, minimizarlo y utilizarlo como herramienta de retención.

El plantear propuestas de empleo acordes a las tendencias del mercado, como lo sugiere Golik (2013), sin duda facilitará la incorporación efectiva de esta generación y con ello contar con una herramienta más para contribuir a la reducción de la rotación de personal, al ampliar la integración e impulsar una adecuada inclusión en las empresas turísticas.

Por último, los resultados obtenidos alimentan la reflexión de que los centennials manifiestan sin limitaciones sus expectativas laborales, lo que invita a repensar la oferta ideal en las empresas turísticas. Los estudios de Espinoza y Toscano (2020), por ejemplo, confirman que el salario emocional es perfectamente compatible con estos trabajadores y que, si es gestionado de manera adecuada, el tan anhelado equilibrio de vida personal y profesional puede ser posible en un sector tan controversial.

\section{Referencias}

Adams, J. S. (1963). Towards an understanding of inequity. Journal of Abnormal Psychology, 67(5), 422-436. https://doi.org/10.1037/h0040968

Adams, J. S. (1965). Inequity in social exchange. Advances in Experimental Social Psychology, 2, 267-299. https://doi.org/10.1016/S0065-2601(08)60108-2

Alderfer, C. P. (1969). An empirical test of a new theory of human needs. Organizational Behavior and Human Performance, 4(2), 142-175. https://doi. org/10.1016/0030-5073(69)90004-X 
Al-Emadi, A., Schwabenland, C. y Wei, Q. (2015). The vital role of employee retention in human resource management: a literature review. The IUP Journal of Organizational Behavior, 14(3), 7-32. https://bit.ly/3j5QPuC

Ato, M., López, J. y Benavente, A. (2013). Un sistema de clasificación de los diseños de investigación en psicología. Anales de Psicología, 29(3), 1038-1059. https:// bit.ly/3nXzelT

Barbero, M. I. (2013). Introducción básica al análisis factorial. Universidad Nacional de Educación a Distancia.

Bennett, J., Pitt, M. y Price, S. (2012). Understanding the impact of generational issues in the workplace. Facilities, 30(7-8), 278-288. https://doi. org/10.1108/02632771211220086

Bialik, K. y Fry, R. (2019, 14 de febrero). Millennial life: how young adulthood today compares with prior generations. Pew Research Center. https://pewrsr. $\mathrm{ch} / 2 \mathrm{~T} 3 \mathrm{WNSa}$

Bizzi, L. (2017). Should HR managers allow employees to use social media at work? Behavioral and motivational outcomes of employee blogging. The International Journal of Human Resource Management, 31(10), 1285-1312. https:// doi.org/10.1080/09585192.2017.1402359

Boschma, J. y Groen, I. (2008). Generación Einstein: más listos, más rápidos y más sociales. Comunicar con los jóvenes del siglo XXI. Gestión 2000.

Bosch, O., Revilla, M. y Paura, E. (2019). Do millennials differ in terms of survey participation? International Journal of Market Research, 61(4). https://doi. org/10.1177/1470785318815567

Bussin, M. y Brigman, N. (2019). Evaluation of remuneration preferences of knowledge workers. SA Journal of Human Resource Management, 10. https://doi. org/10.4102/sajhrm.v17i0.1075

Carter, T. y Walker, W. (2018). Retaining, cultivating, and investing strategies for leading millennial managers. The Journal of Government Financial Management, 67(2), 20-25. https://bit.ly/2HdlTtl

Cloninger, S. (2002). Teorías de la personalidad. Pearson - Prentice Hall.

Costanza, D. P., Badger, J. M., Fraser, R. L., Severt, J. y Gade, P. (2012). Generational differences in work-related attitudes: a meta-analysis. Journal of Business and Psychology, 27, 375-394. https://doi.org/10.1007/s10869-012-9259-4 
Ertas, N. (2015). Turnover intentions and work motivations of millennial employees in federal service. Public Personnel Management, 44(3), 401-423. https://doi. org/10.1177/0091026015588193

Espinoza, A. y Toscano, J. A. (2020). Salario emocional: una solución alternativa para la mejora del rendimiento laboral. Nova Rua, 12(20), 72-89. http://dx.doi. org/10.20983/novarua.2020.20.5

Espinoza, C. y Ukleja, M. (2016). Managing the millennials: discover the core competencies for managing today's workforce. Wiley.

Ferri-Reed, J. (2014). Are millennial employees changing how managers manage? The Journal for Quality \& Participation, 37(2), 15-35. https://bit.ly/2T8qOjr

Fischer, L. y Espejo, J. (2018). Are young university millennials compulsive in their purchase? Horizonte Sanitario, 17(3), 189-196. https://doi.org/10.19136/ hs.a17n3.2244

George, J. y Wallio, S. (2017). Organizational justice and millennial turnover in public accounting. Employee Relations, 39(1), 112-126. https://doi.org/10.1108/ER11-2015-0208

Gibson, J., Greenwood, R. y Murphy, E. (2009). Generational differences in the workplace: personal values, behaviors, and popular beliefs. Journal of Diversity Management, 4(3), 1-7. https://doi.org/10.19030/jdm.v4i3.4959

Golik, M. (2013). Las expectativas de equilibrio entre vida laboral y vida privada y las elecciones laborales de la nueva generación. Cuadernos de Administración, 26(46), 107-133. https://bit.ly/3ka9CGu

González, R. (2011). La incorporación de la Generación Y al mercado laboral. El caso de una entidad financiera de la ciudad de Resistencia. Palermo Business Review, 5(1), 67-93. https://bit.ly/2IDBW6a

Guillot-Soulez, C. y Soulez, S. (2014). On the heterogeneity of Generation Y job preferences. Employee Relations, 36(4), 319-332. https://doi.org/10.1108/ER-072013-0073

Hair, J., Anderson, R., Tatham, R. y Black, W. (1999). Análisis multivariante. Prentice Hall Iberia.

Haserot, P. (2009). The keys to maintaining professionalism and harmony across the generations at work. The Professional Lawyer, 19(3), 3-5, 17. 
Herbison, G. y Boseman, G. (2009). Here they come - Generation Y. Are you ready? Journal of Financial Service Professional, 63(3), 33-34. https://bit.ly/2Hd0AKd

Hershatter, A. y Epstein, M. (2010). Millennials and the world of work: an organization and management perspective. Journal of Business \& Psychology, 25, 211223. https://doi.org/10.1007/s10869-010-9160-y

Herzberg, F. y Mausiner, B. S. (1959). The motivation to work. Wiley.

Holland, P., Cooper, B. y Hecker, R. (2016). Use of social media at work: a new form of employee voice? The International Journal of Human Resource Management, 27(21), 2621-2634. https://doi.org/10.1080/09585192.2016.1227867

Jonsen, K., Weg, S. y Martin, R. (2012). Cómo gestionar a los nativos digitales. Capital Humano, 266, 60-65. https://bit.ly/3IVDUNq

Kahn, W. (2017). Psychological conditions of personal engagement and disengagementat work.Academy ofManagement,33(4). https://doi.org/10.5465/256287

Karriker, J. y Hartman, N. (2018). Social media and dynamic capabilities: mining millennial resources. Journal of Organizational Psychology, 18(4), 43-56. https:// doi.org/10.33423/jop.v18i4.84

Kelly Mercadotenia. (2017, 15 de mayo). Índices de rotación a nivel nacional. https:// bit.ly/3m7spTt

Kowske, B., Rasch, R. y Wiley, J. (2010). Millennials' (lack of) attitude problem: an empirical examination of generational effects on work attitudes. Journal of Business and Psychology, 25(2), 265-279. https://doi.org/10.1007/s10869-0109171-8

Kultalahti, S. y Viitala, R. (2015). Generation Y - Challenging clients for HRM. Journal of Managerial Psychology, 30(1), 101-114. https://doi.org/10.1108/JMP-082014-0230

Kuron, L., Lyons, S., Schweitzer, L. y Ng, E. (2015). Millennials' work values: differences across the school to work transition. Personnel Review, 44(6), 991-1009. https://doi.org/10.1108/PR-01-2014-0024

Locke, E. A., Latham, G., Smith, K. y Wood, R. (1990). A theory of goal setting and task performance. Prentice Hall.

Madero, S. y De la Garza, J. (2017, junio). Validación del modelo de las prácticas de atracción y retención laboral desde la perspectiva del trabajador joven. Gestión de la innovación para el desarrollo sostenible de los negocios 
[ponencia]. 3er. Congreso Internacional de Investigación en Escuelas y Facultades de Negocios, Nuevo León, México.

Madero, S., Díaz, P. y Núñez, F. (2016, mayo). Análisis exploratorio de las prácticas de atracción y retención en ambientes de trabajo desde la perspectiva de la Generación Y. Gestión social: organizaciones humanas para una sociedad incluyente [ponencia]. XX Congreso Anual de la Academia de Ciencias Administrativas AC (Acacia), Yucatán, México.

Magallón, R. (2016). El ADN de la Generación Z. Entre la economía colaborativa y la economía disruptiva. Revista de Estudios de Juventud, 114, 29-44. https://bit. ly/2lyJRS1

Maslow, A. (1943). A theory of human motivation. Psychological Review, 50(4), 370396. https://doi.org/10.1037/h0054346

McClelland, D. C. (1961). The achieving society. Van Nostrand.

McGregor, D. (1985). The human side of enterprise. McGraw-Hill Education.

Meister, J. (2012, 5 de octubre). Tree reasons you need to adopt a millennial mindset regardless of your age. Forbes. https://bit.ly/2H6Y87T

Meza, A. (2019, 20 de marzo). Rotación de personal: cinco tips para reducirla. Forbes. https://bit.ly/2T3D3he

Moreno, L., López, V. y Marín, M. (2015). Comportamiento de la tasa de rotación laboral en la industria maquiladora en Mexicali, Baja California, 2009 - 2013. Revista Global de Negocios, 3(4), 11-26. https://bit.ly/37gcBt1

Murillo, E. (2017). Attitudes toward mobile search ads: a study among mexican millennials. Journal of Research in Interactive Marketing, 11(1), 91-108. https:// doi.org/10.1108/JRIM-06-2016-0061

Ng, E., Schweitzer, L. y Lyons, S. (2010). New generation, great expectations: a field study of the Millennial Generation. Journal of Business and Psychology, 25, 281-292. https://doi.org/10.1007/s10869-010-9159-4

Paradinas, M. C. (2020). La gestión del conflicto en el ámbito laboral de las organizaciones turísticas: una herramienta de marketing interno. Holos, 1, 1-12. https://doi.org/10.15628/holos.2020.8260

Parry, E. y Urwin, P. (2017). The evidence-base for generational differences: where do we go from here? Work, Aging and Retirement, 3(2), 140-148. https://doi. org/10.1093/workar/waw037 
Rani, N. y Samuel, A. (2016). A study on generational differences in work values and person-organization fit and its effect on turnover intention of generation Y in India. Management Research Review, 39(12), 1695-1719. https://doi. org/10.1108/MRR-10-2015-0249

Rani, S. y Mukherjee, S. (2017). Development of a causal framework linking work values, perceived organisational support, and job attitudes. Journal of Strategic Human Resource Management, 6(3), 6-16. https://bit.ly/31e7uWA

Robbins, S. (1999). Comportamiento organizacional. Pearson - Prentice Hall.

Schultz, D. (1992). Psicología industrial. McGraw-Hill.

Smith, T. (2010). Work-life balance perspectives of marketing professionals in Generation Y. Services Marketing Quarterly, 31(4), 434-447. https://doi.org $/ 10.1080 / 15332969.2010 .510724$

Solnet, D., Kralj, A. y Kandampully, J. (2012). Generation Y employees: an examination of work attitude differences. Journal of Applied Management and Entrepreneurship, 17(3), 36-54. https://bit.ly/301Muwb

Thompson, C. y Gregory, J. B. (2012). Managing millennials: a framework for improving attraction, motivation, and retention. The Psychologist-Manager Journal, 15(4), 237-246. https://doi.org/10.1080/10887156.2012.730444

Torrado, S. (2015, 31 de enero). Empresas para los jóvenes. Latin Trade Magazine. https://bit.ly/3jONH2Y

Twenge, J. (2010). A review of the empirical evidence on generational differences in work attitudes. Journal of Business and Psychology, 25, 201-210. http://dx.doi. org/10.1007/s10869-010-9165-6

Tyler, K. (2013). Organizational and employee development: new kids on the block. HR Magazine, 59(10), 34-40.

U.S. Department of Education. (2005). What is scientifically based research? A guide for teachers. https://bit.ly/3IZI8F7

Ventura-León, J. L. y Caycho-Rodríguez, T. (2017). El coeficiente Omega: un método alternativo para la estimación de la confiabilidad. Revista Latinoamericana de Ciencias Sociales, Niñez y Juventud, 15(1), 625-627. https://bit.ly/2HgE2s4

Vroom, V. (1994). Work and motivation. Wiley.

Wilton, P. (2008). Unlocking the talent of Generation Y. Engineering \& Technology, 3(14), 80-83. http://dx.doi.org/10.1049/et:20081421 